\title{
Fast and Accurate Method for Identifying High-Quality Protein-Interaction Modules by Clique Merging and Its Application to Yeast
}

\section{Supplement Materials}

Chi Zhang, Song Liu, Yaoqi Zhou*

Howard Hughes Medical Institute Center for Single Molecule Biophysics, Department of Physiology \& Biophysics, State University of New York at Buffalo, 124 Sherman Hall, Buffalo, NY 14214

${ }^{*}$ Corresponding Author: Dr. Yaoqi Zhou, Howard Hughes Medical Institute Center for Single Molecule Biophysics and Department of Physiology \& Biophysics, State University of New York at Buffalo, 124 Sherman Hall, Buffalo, NY 14214, Phone: (716) 829-2985, Fax: (716) 829-2344, Email: yqzhou@buffalo.edu

\section{$\mathrm{MC}^{2}$ Algorithm}

(1) Enumerate all cliques, $C$; //Using Algorithm 457.

$/ /$ merge cliques (size $>=4$ )

(2) $c_{m}=$ the biggest one in all non-merged cliques;

(3) if (size_of $\left.\left(c_{m}\right)<3\right)$ break;

(4) for $c_{j} \in C$ do

$$
c n_{j}=\text { number_of_common_node }\left(c_{j}, c_{m}\right) \text {; }
$$

(5) for each $c n_{j}$ in descending order do

$$
\begin{aligned}
& \text { if }\left(c n_{j} / \max \left(c n_{k}, k=0 . . n\right)>\right.\text { cut_off_1 and } \\
& \left.Q\left(c_{j} \cup c_{m}\right)>\text { cut_off_2 }\right) \\
& c_{m}=c_{j} \cup c_{m} ;
\end{aligned}
$$

(6) for each non-merged cliques $c_{j}$,

$$
c_{j}=c_{j}-\left(c_{j} \cap c_{m}\right) \text {; }
$$

(7) save $c_{m}$ as a module, and $c_{m}$ is set to be merged;

(8) goto step (2).

//deal with orphan cliques

(9) for each orphan cliques $c_{i}$ do, for each module $M_{j}$ do

find the module $M_{j}$ that $Q_{i j}=Q\left(c_{i} \cup M_{j}\right)$ is maximum;

END.

$$
\begin{aligned}
& \text { if }\left(k_{i j}^{i n}>k_{i j}^{\text {out }} \text { and } Q_{i j}>\text { cut_off_3 }\right) \\
& \quad M_{j}=M_{j} \cup c_{i} ;
\end{aligned}
$$

Note: cut_off_1=cut_off_ $2=r_{\text {cutoff }}$ and cut_off_ $3=Q_{\text {cutoff }}^{\text {orphan }}$. 


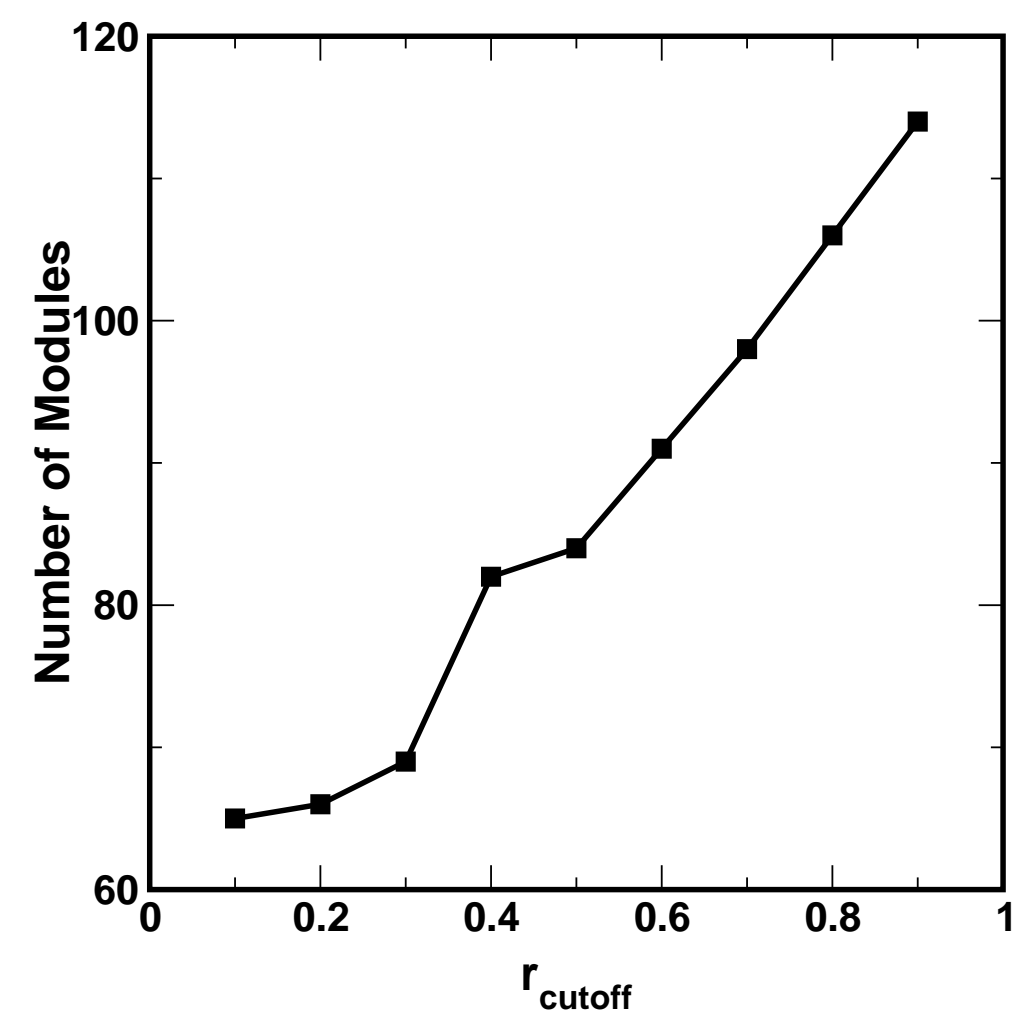

Fig. 1. The number of modules as a function of the key cutoff value $\left(r_{\text {cutoff }}\right)$.

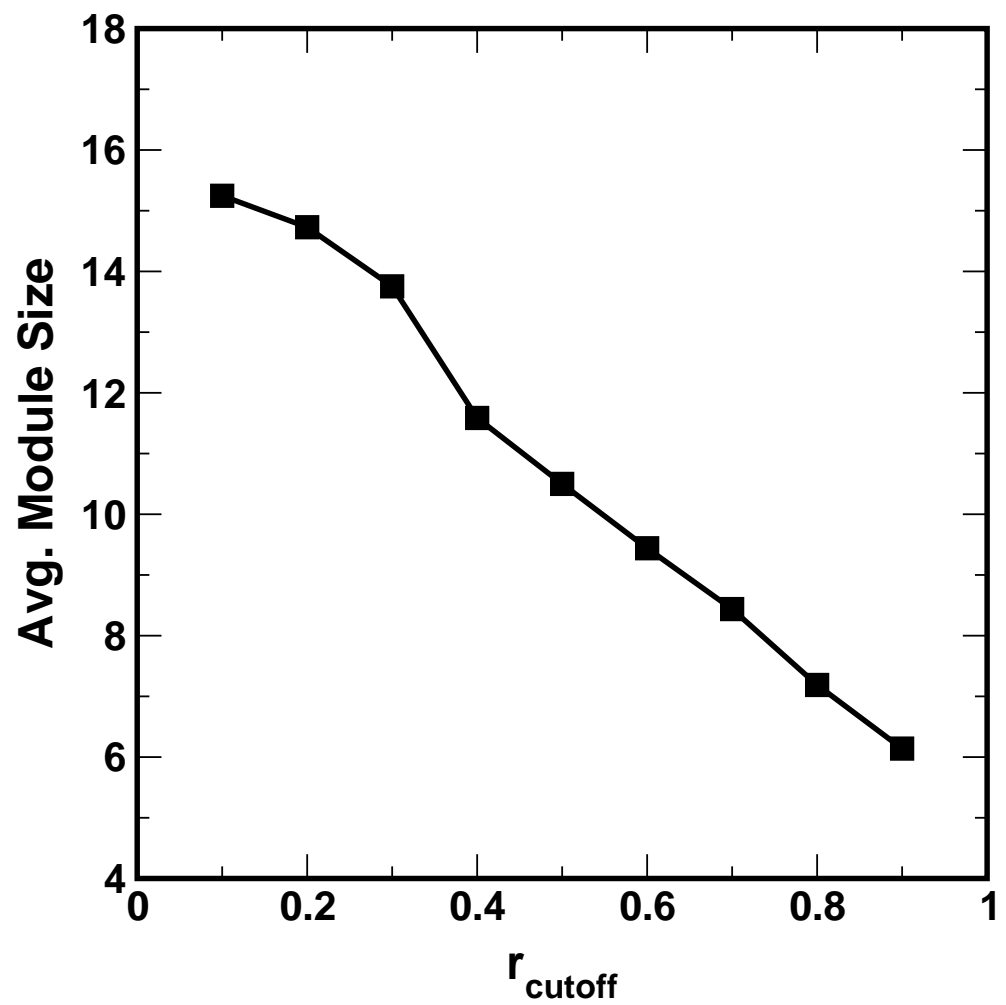

Fig. 2. As in Fig.1 but for the average number of proteins per module. 


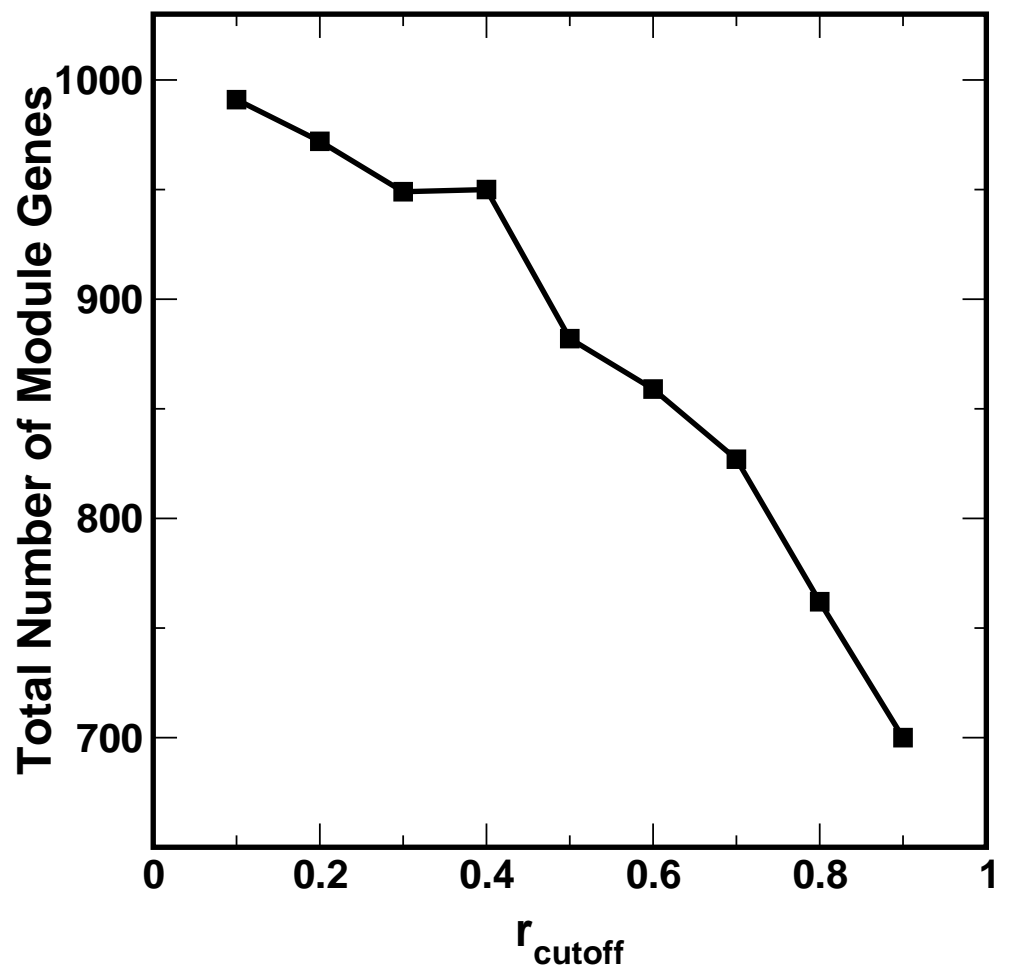

Fig. 3. As in Fig.1 but for total number of genes in predicted modules.

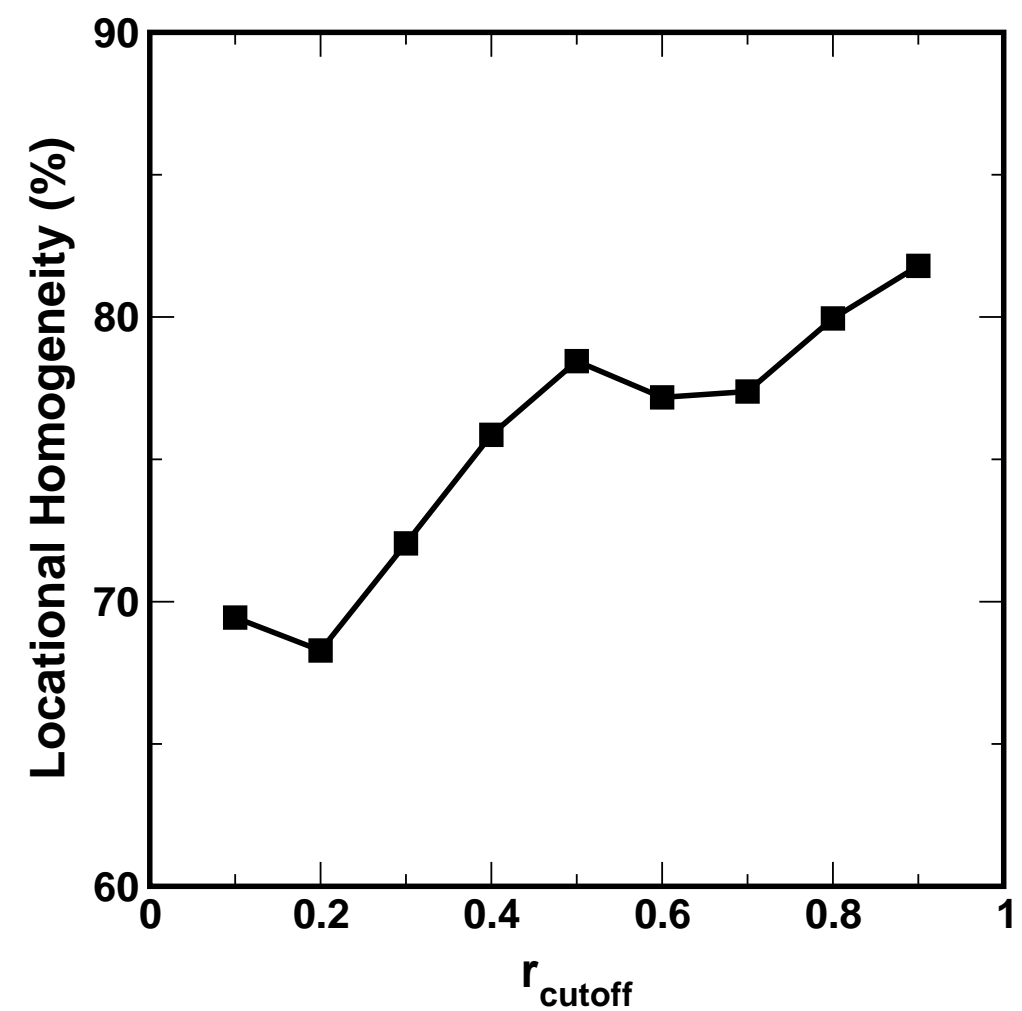

Fig. 4. As in Fig.1 but for the average locational homogeneity of predicted modules. 


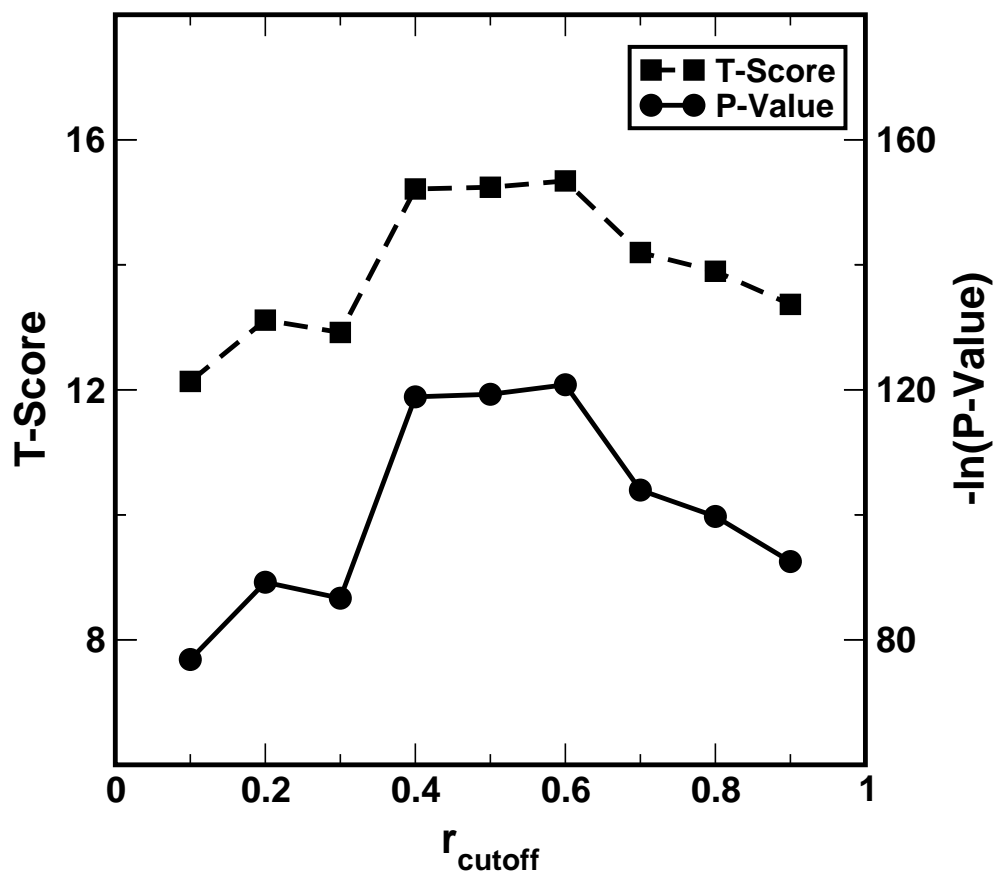

Fig. 5. As in Fig.1 but for the significance (T-Score and P-value) of enrichment of co-expressed genes in predicted modules over the whole network graph. 